\title{
RESEARCH ON INTEGRATED TEMPERATURE SENSOR BASED ON LOW POWER SINGLE CHIP
}

\author{
Li Xiao-xiong ${ }^{l}$ Xu Wei-min ${ }^{1}$, Mohammad Hadi Nematollahi ${ }^{1}$ \\ ${ }^{1}$ Yellow River Conservancy Technical Institute, Kaifeng, Henan 475003, China
}

\begin{abstract}
To effective use of wind energy resources in low wind region, the "vertical axis-building block -pressure type" blade is developed. Firstly, a large rectangular frame is designed in order to form a slice of whole blades; Secondly, several small units which can turn freely around the shaft are installed on the whole blades; Finally, the two pieces of the overall blades are made of a pair of big blade and it is installed in the axis of wind turbine, which is symmetrical of left and right sides. While working, wind energy would press vertically a whole blade to get moving torque and drive the generator to power. To verify the design results, this article made a small physical device and tested under different wind speed. The results showed that the device started low wind speed performance was good and operation was stable and reliable.
\end{abstract}

Keywords: The "Vertical Axis-Building Block-Pressure Type" Blade, Whole Blades; Unit Small Blade, The Use Ratio of Wind Energy.

\section{Introduction}

Temperature is a physical parameter that people are exposed to most frequently in daily life, and it is also one of the most sensitive information for people. Throughout the history of quantitative research on temperature, quantitative tools can be divided into two categories: "thermometer" and "temperature sensor". In fact, the quantitative study of temperature began in the 16th century. The world's first air thermometer used to measure temperature was invented by the famous scientist Galileo Galilei in 1593.This thermometer is the prototype of the thermometer that is now widely used. Ferdinand in 1654 , Galileo's students on the basis of the value of their teacher developed alcohol thermometer, five years after the French astronomer in the produced measurable high temperature of $357^{\circ} \mathrm{C}$ and for the first time $-39^{\circ} \mathrm{C}$ temperature mercury thermometer. In the years that followed, various thermometers were developed by scientists. In 1821, German physicists first opened the door to turning temperature into electrical signals [1]. With the discovery of the first thermoelectric effect, it made the world's first true temperature sensor -thermocouple sensor, which brought the quantitative research on temperature into the stage of temperature sensor and thus kicked off the development of temperature sensor. It was these two landmark experiments that led to the quantitative study of temperature.

In this paper, the system architecture and circuit design of low-power monolithic integrated temperature sensor is taken as the main work content to study and realize a precision adjustable time-domain integrated temperature sensor combined with the working characteristics of voltage domain. Improve the stability of time domain integrated temperature sensor to power supply voltage and process change technology, improve the linearity of temperature sensor output signal technology.

\section{Literature Review}

Bhandari, a. s. and Jamal, f. i. et al. put forward A thermoelectric element design theory that the fabrication process is compatible with the integrated circuit process [2-3]. Two years later, Jamal, f. i proposed and successfully developed the world's first integrated temperature sensor chip [4]. However, the first integrated temperature sensor was not introduced until the 1980s.Subsequently, a large number of integrated temperature sensors have been developed with more and more functions and better performance. Among them, the wellknown integrated temperature sensor manufacturers include ADI, ST, TI, Microchip, MAXIM and NXP, etc. Their products are still widely used in industry, medical equipment, computers and consumer products. In 2008, Zhao, n., a famous professor in the field of integrated temperature sensors, presented a report on "designing intelligent temperature sensors in standard CMOS processes" at the IEEE International Electron Devices Meeting. In the speech, he mentioned that "the integration of temperature sensor in standard CMOS process will bring the advantages of cost reduction, size reduction, robustness improvement and rich functionality to integrated circuit chips" [5].

The development of temperature sensors can be divided into two categories: discrete temperature sensors and integrated temperature sensors. The former USES thermocouple, thermistor and other 
elements as the temperature sensing part. With the development of research, new materials and films have been developed to fabricate these temperature sensing elements to improve the performance of temperature sensors. After years of development, the discrete temperature sensor has higher measurement accuracy and wider temperature measurement range, and has excellent stability, so it is widely used in agriculture, industry, military and other fields of large equipment. In recent years, the rapid development of integrated circuits, integrated circuit manufacturing level progress, so that more and more functional modules can be integrated into the same chip. These new integrated circuits not only reduce the cost of using products, but also improve the portability of products. However, with the increasing number of components per unit area of the chip, the heat generated by the chip is also increasing, increasing the potential for device performance degradation or even failure, thus triggering people's attention to chip temperature monitoring and management. Although the discrete temperature sensor can help people to realize the measurement and control of temperature, its large volume and incompatible with the manufacturing process of integrated circuit make it impossible to be applied in this situation [6]. In addition, the advent of wearable devices has made cheap and portable temperature sensors popular. It is the demand above that motivates people to research and develop miniaturized temperature sensor -- integrated temperature sensor.

\section{Low Power Consumption Monolithic Integrated Temperature Sensor Analysis and Simulation Test}

Up to now, it has been half a century since Alirezaei, i. s. proposed and successfully developed the world's first integrated temperature sensor in 1966 [7]. However, the idea of using the characteristic of PN junction voltage changing with temperature to realize the temperature induction of integrated circuit is still used up to now, and forms an important branch of integrated temperature sensor - integrated temperature sensor in voltage domain. Another important branch of integrated temperature sensor was started in 2005 by a team led by professor Poki Chen.

For the first time, the temperature sensing method based on the temperature characteristics of the delay time of the circuit unit in the integrated circuit was proposed, and a time domain integrated temperature sensor was developed.

\subsection{Voltage field integrated temperature sensor}

The voltage domain type integrated temperature sensor is composed of a temperature sensing circuit, a reference signal generation circuit and an analog- digital signal converter (ADC). The specific working principle of such integrated temperature sensor is as follows:

1) The temperature sensor circuit monitors the temperature of the measured object and converts the temperature into an analog voltage (or current) that is positively or negatively correlated with it. BJT is often used to construct the core modules of temperature sensing circuits as a device that provides voltages Proportional to absolute temperature (PTAT) and Complementary to absolute temperature (CTAT) [8].

2) The reference signal generation circuit provides a reference voltage (or reference current) that does not change with the temperature for the $\mathrm{ADC}$ in this type of integrated temperature sensor. Since the quantization of ADC is based on the comparison between the temperature information output by the temperature induction circuit and the reference signal, the signal needs to have good temperature stability, as well as good resistance to process and power supply voltage changes. Bandgap circuits are usually used to generate reference signals [9-10].

3) The ADC compares the PTAT voltage (or CTAT voltage) of the output of the temperature sensing circuit representing the temperature of the measured object with the reference voltage provided by the reference signal generation circuit, and quantifies the output temperature information of the temperature sensing circuit based on the comparison result, and finally converts it into the digital code representing the temperature. The digitized temperature information can be either given to the digital processor for processing or can be directly read by human beings through encoding and other operations [11].

An integrated temperature sensor based on time domain quantization is proposed. The sensor USES FDC composed of a counter and an encoder to convert the frequency signal representing the temperature information output by the temperature controlled oscillator (TCO). TCO is composed of a temperature-sensing circuit with PTAT current output and an AFC composed of a relaxation oscillator. AFC is responsible for converting the current signal output from the temperature induction circuit into the corresponding frequency signal input to the counter of FDC.

The counter quantifies the PTAT frequency of AFC output by using the reference frequency of the relaxation oscillator with the same structure as AFC, and converts the quantized result into digital temperature information in the encoder.

Therefore, the accuracy of the integrated temperature sensor can be adjusted by changing the reference clock frequency provided to the counter, as shown in equation (1).

$$
\text { Re solution }=\left(\frac{\Delta f_{T E M P}}{\Delta T \cdot f_{R E F}}\right)^{-1}
$$

According to the oscillation period expression of relaxation oscillator based on DT technology and SR 
technology, when the oscillator is used as AFC in the integrated temperature sensor mentioned, and considering two different cases of resistance

CASE I is: temperature characteristics in the oscillator circuit, the frequency of its output representing temperature information can be expressed as follows:

CASE II is :

$$
f_{F T A T(C A S E I)}=\left(\Phi_{C T A T}\right)^{-1}=\frac{I_{c(P T A T)}}{2 C\left|I_{2(R E F)} R_{1}-I_{1(R E F)} R_{2}\right|}
$$

$$
f_{P T A T(C A S E I I)}=\left(\Phi_{C T A T}\right)^{-1}=\frac{I_{C(P T A T)}}{2 C\left[I_{2(R E F)} R_{1}+\left(I_{1(R E F)}+I_{2(R E F)}\right) R_{2}\right]}
$$

where, $\mathrm{C}$ is the capacitance of the timing capacitor; $\mathrm{R} 1$ and $\mathrm{R} 2$ are the resistance values of the timing resistor; Ix(REF) $(x=1,2)$ represents the current generated by the comparator flipping threshold voltage with temperature invariant characteristics; IC(PTAT) represents the charging current of a timing capacitor with PTAT characteristics. In the circuit design of low-power monolithic integrated temperature sensor, whether it is used as an AFC or a relaxation oscillator as a reference clock source,

$$
\text { Re solution }=\left(\frac{\Delta f_{T E M P}}{\Delta T \cdot f_{R E F}}\right)^{-1}=\left(\frac{\Delta f_{P T A T}}{\Delta T \cdot f_{R E F}}\right)^{-1}=\frac{\Delta T \cdot I_{C(R E F)}}{\Delta I_{C(P T A T)}}
$$

Among them, the $\triangle \mathrm{T}$ said temperature variation; $\triangle \mathrm{IC}(\mathrm{PTAT})$ as the AFC PTAT caused by $\Delta \mathrm{T}$ timing capacitor in the charging current variation; IC(REF) refers to the charging current of the timing capacitor in the reference clock circuit that does not change with the temperature. Equation (4) shows that the changes of component values such as resistance and capacitance caused by IC fabrication process will not affect the temperature resolution of the proposed integrated temperature sensor, thus enhancing the ability of the temperature sensor to resist process changes. The temperature measurement error of the integrated temperature sensor is determined by the linearity of PTAT current IC (PTAT) with the required current IC, I 1 and I 2 are provided by the temperature sensing circuit.

Therefore, according to the expression of the temperature resolution of the low-power monolithic integrated temperature sensor based on timedomain quantization proposed in this paper, the temperature resolution is only related to the ratio of charging current of the reference clock circuit and the timing capacitor in AFC, specifically temperature in AFC and the temperature stability of reference current IC(REF) in reference clock circuit. However, the temperature measurement error is essentially still determined by the temperature linearity of the PTAT current output by the temperature induction circuit and the temperature stability of the reference current. It can be seen that the proposed integrated temperature sensor not only avoids the ADC design with high precision and low quantization error, but also solves the problem that the precision and temperature detection range of the traditional time-domain integrated temperature sensor are limited by the "gate" circuit structure.
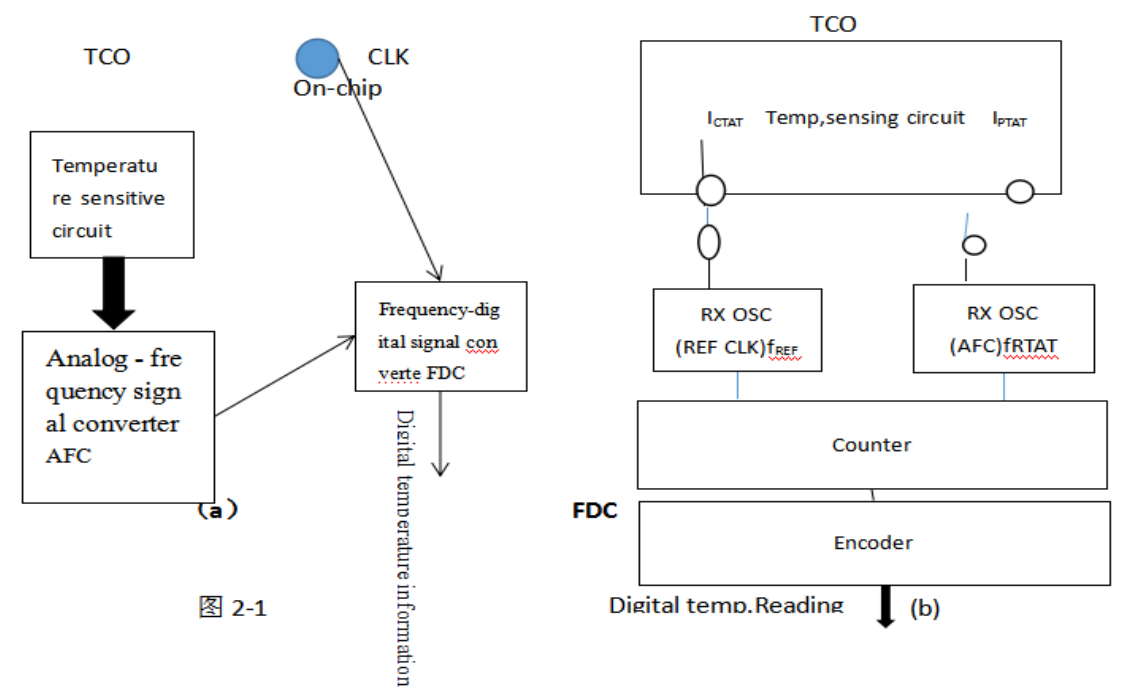

Figure 1: Conversion flow chart 


\subsection{Time domain integrated temperature sensor}

The basic working idea of the time-domain type integrated temperature sensor is: first, convert the temperature to be measured into time information, then quantify the obtained time, and convert the corresponding digital code according to the length of the time. Compared with the voltage domain integrated temperature sensor, the time domain integrated temperature sensor is somewhat obscure and difficult to understand, so it came out relatively late. However, the module circuit of such integrated temperature sensor can be realized by "gate" circuit, which is convenient for full digital integration.

Therefore, after its architecture was born, it quickly attracted many scholars to join its research ranks and became an important branch in the development process of integrated temperature sensor.

System structure of the temperature sensor and voltage domain types are very similar, but this kind of temperature sensor based on Time domain, and the system clock reference signal at the same Time, therefore, need not - digital signal converter circuit to generate extra Time (Time - to - digital converter, TDC) needed for the reference signal, simplify the circuit design. In addition, the digital "gate" circuit structure is also one of the characteristics of this kind of integrated temperature sensor.

The traditional time-domain integrated temperature sensor works according to the following principles:

1) When the temperature sensor system starts running, the temperature sensor circuit will receive the Start signal Start. After passing through the delay line composed of delay units, the signal is obtained by "xor" operation with itself. As the delay time of the delay unit will increase with the increase of temperature, the width of the pulse obtained through the above "xor" operation will also change with the change of temperature, thus realizing the conversion of temperature information to time signal.

2) The TDC quantifies the pulse width of the output of the temperature induction circuit according to the given reference clock, and encodes the quantized result and outputs it, thus obtaining the digitized temperature information.

The quantization of pulse width by TDC can be completed by counter. Therefore, compared with voltage domain integrated temperature sensor using ADC as converter, the circuit design of time domain integrated temperature sensor is relatively simple.

The low-power monolithic integrated temperature sensor based on time-domain quantization was finally designed and manufactured into a standard CMOS process at $180 \mathrm{~nm}$.In order to facilitate testing and troubleshooting, the FDC composed of a counter and an encoder in the integrated temperature sensor is designed and implemented in the field programmable gate array test board. Since the reference clock circuit and AFC in the integrated temperature sensor are designed with double power sources (VDD and VDD_DT\&SR), the power consumption of the temperature sensor should be composed of the power consumed on the two power sources respectively.

The experimental results show that the system power supply VDD with voltage of $1.2 \mathrm{~V}$ consumes A total current of $15.64 \mu \mathrm{A}$ without taking into account the power consumption of FDC and output buffer. The "DT and SR" branch power supply with voltage of $0.75 \mathrm{~V}$ consumes A total current of $17.86 \mu \mathrm{A}$.FDC circuit realized by FPGA consumes current 98nA (RMS) at $1.2 \mathrm{~V}$ working voltage.

\section{Test Analysis}

\subsection{Voltage field integrated temperature sensor}

Table 1 shows the temperature and voltage characteristics of polycrystalline resistance and capacitor elements provided by the IC fabrication process used to design the low-power monolithic integrated temperature sensor proposed in this paper. It can be seen that the polycrystalline resistance provided by this process has the same temperature characteristics, which corresponds to CASE I.

Considering that the specific selection of resistance value and current value in this paper makes $\mathrm{R} 1 \cdot(\mathrm{I} 1+\mathrm{I} 2)<\mathrm{I} 1 \cdot(\mathrm{R} 1+\mathrm{R} 2)$, the flip threshold voltage of the comparator in the relaxation oscillator is fixed.

Table 1. Characteristics of resistance and capacitance in process of low-power monolithic integrated temperature sensor based on timedomain quantization

\begin{tabular}{|c|c|c|c|}
\hline & Rppoly & Rnpoly & C \\
\hline TC1 & $\begin{array}{c}-1.237^{*} 10- \\
4 / 0 C\end{array}$ & $\begin{array}{c}1.232^{*} 10- \\
3 / 0 C\end{array}$ & $\begin{array}{c}-2.743^{*} 10- \\
5 / 0 C\end{array}$ \\
& & $2.035^{*} 10-$ & 0 \\
TC2 & $0.6^{*} 10-$ & $6 / 0 \mathrm{C}$ & \\
& $6 / 0 \mathrm{C}$ & $1.114^{*} 10-$ & $2.363^{*} 10-$ \\
VC1 & $3.658^{*} 10-$ & $5 / \mathrm{V}$ & $5 / \mathrm{V}$ \\
& $5 / \mathrm{V}$ & $4.4 * 10-$ \\
VC2 & $-3.426^{*} 10-$ & $-1.99^{*} 10-$ & $1.4^{*}$ \\
& $4 / \mathrm{V}$ & $3 / \mathrm{V}$ & $5 / \mathrm{V}$ \\
\hline
\end{tabular}

Compared with the rest of the module circuit, as the proposed integrated temperature sensor AFC and reference clock relaxation oscillator has the highest power consumption, therefore, in order to meet the power requirements in the system index, relaxation oscillator to be optimized design. 


\subsection{Time domain integrated temperature sensor}

Figure 2 shows the simulation results and measured results of AFC output frequency designed in this paper. As can be seen from the figure, the measured output frequency of the circuit is slightly higher than that of the typical process Angle in the simulation.

In addition, the simulation results show that although the design of an AFC circuit under different process Angle, differ from that of the output

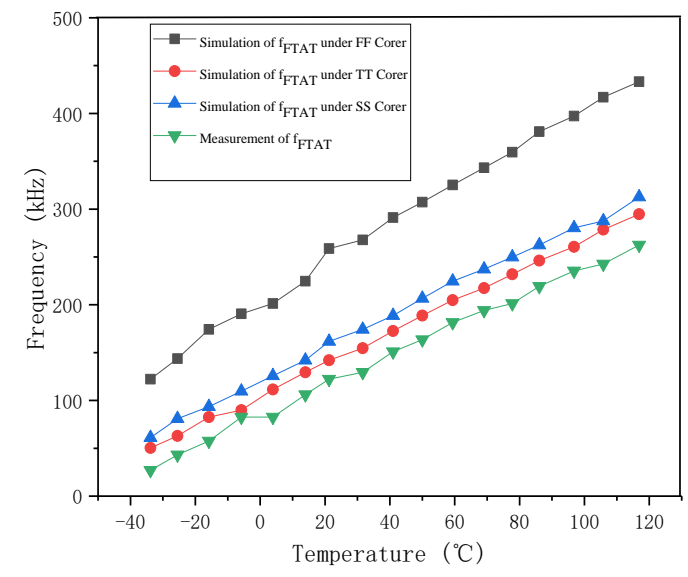

frequency range of beginning and ending, but the output frequency of the slope in the whole temperature range (to $-40^{\circ} \mathrm{C}$ to $120^{\circ} \mathrm{C}$ ) changes in quantity are less than $\pm 4.2 \%$ (TT and SS Angle of process variation is less than $\pm 4 \%$ ). However, the slope change of the output frequency of the circuit obtained from the measured results is worse. In $30^{\circ} \mathrm{C}$ to $120^{\circ} \mathrm{C}$ (due to the temperature test box can only work to $-30^{\circ} \mathrm{C}$ ) temperature range, the measured AFC output frequency of the maximum slope variation of $4.5 \%$.

Figure 2: Analysis of frequency measurement results

The actual performance of the monolithic integrated temperature sensor is summarized in Table 2. In order to facilitate the comparison, the performance parameters of the time-domain integrated temperature sensor that have been publicly reported in recent years are also included in the table.

Where, the high quality factor Fo M is defined as "Power consumption temperature Resolution conversion rate", that is, Fo $M=$ (Power $\times$ Resolution)/fs.

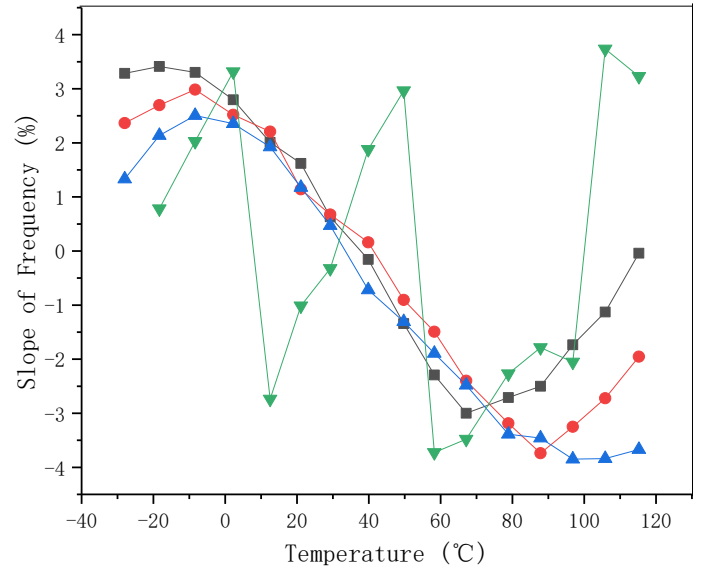

It can be seen from this that except for the temperature measurement error failing to meet the initial set system index requirements, the rest of the performance parameters have met the system index requirements.

In addition, compared with other research results, the integrated temperature sensor based on time-domain quantization proposed in this paper not only realizes on-chip integration of reference clock circuit, but also has the lowest power consumption.

Table 2. Comparisons of relevant parameters

\begin{tabular}{|c|c|c|c|c|c|c|c|c|}
\hline Ref. & $\begin{array}{c}\text { Tech. } \\
(\mathrm{nm})\end{array}$ & $\begin{array}{c}\text { Area } \\
(\mathrm{mm} 2)\end{array}$ & $\begin{array}{c}\mathrm{Fs} \\
(\mathrm{kHz})\end{array}$ & $\begin{array}{c}\text { Power } \\
(\mu \mathrm{W})\end{array}$ & $\begin{array}{c}\text { Resoiution } \\
\left({ }^{\circ} \mathrm{C}\right)\end{array}$ & $\begin{array}{c}\text { Inaccuracy } \\
\left({ }^{\circ} \mathrm{C}\right)\end{array}$ & $\begin{array}{c}\text { Calibration } \\
\text { Method }\end{array}$ & Fo M \\
\hline$[34]$ & 350 & 0.6 & 0.002 & 36.7 & 0.0918 & $\begin{array}{c}-0.25 \sim 0.35 \\
@ 0 \sim 90\end{array}$ & 2 -point & $1.68 \mu{ }^{\circ} \mathrm{C}$ \\
\hline$[41]$ & 180 & 0.18 & 80 & 36 & 0.25 & $\begin{array}{c} \pm 0.5 \\
@ 0 \sim 100\end{array}$ & 1 -point & $113 \mu]^{\circ} \mathrm{C}$ \\
\hline$[50]$ & 130 & 0.12 & 5 & 1200 & 0.28 & $\begin{array}{c} \pm 4 \\
@ 0 \sim 100\end{array}$ & 1 -point & $187 \mu]^{\circ} \mathrm{C}$ \\
\hline$[59]$ & 65 & 0.008 & 469 & 500 & 0.18 & $\begin{array}{c} \pm 1.5 \\
@ 0 \sim 110\end{array}$ & 1 -point & $192 \mu{ }^{\circ} \mathrm{C}$ \\
\hline$[73]$ & 130 & 0.031 & 430 & 288 & 0.595 & $\begin{array}{c}-0.6 \sim 1 \\
@ 20 \sim 120\end{array}$ & 1 -point & $399 \mu]^{\circ} \mathrm{C}$ \\
\hline$[83]$ & 160 & 0.0046 & 0.9 & 3600 & 0.6 & $\begin{array}{c} \pm 1.5 \\
@-10 \sim 125\end{array}$ & 1 -point & $2.4 \mu]^{\circ} \mathrm{C}$ \\
\hline $\begin{array}{c}\text { This } \\
\text { work }\end{array}$ & 180 & 0.236 & 0.5 & 32.3 & 0.5 & $\begin{array}{c}-0.5 \sim 1.5 \\
@-30 \sim 120\end{array}$ & 2 -point & $32.3 \mu \mathrm{J}^{\circ} \mathrm{C}$ \\
\hline
\end{tabular}




\section{Conclusion}

In this paper, the temperature sensor based on lowpower monolithic integration is studied, and two types of temperature sensor are proposed, one is the voltage domain integrated sensor, and the other is the time domain sensor. The simulation tests are carried out on them, from which the improvement and optimization methods are proposed. Although there is an error between the actual measurement and the simulation, it does not affect it. We provide guidance and Suggestions for the follow-up research. With the continuous expansion of the field of temperature monitoring, the functional circuit of temperature sensor has been developed by leaps and bounds, and its market size is increasing day by day. However, with the increasing demand for miniaturization, portability and low-cost equipment, it is increasingly difficult for the large and expensive discrete temperature sensors to meet the market demand, thus resulting in the birth of integrated temperature sensors. In the future, the research on integrated temperature sensing of low-power single chip will be more refined and more breakthroughs will be made.

\section{Reference}

[1] Malits, M., Brouk, I., \& Nemirovsky, Y. (2018). Study of cmos-soi integrated temperature sensing circuits for on-chip temperature monitoring. Sensors, 18(5), 1629-.

[2] Bhandari, A. S., Chaudhuri, A., Roy, S., Negi, S., \& Sharad, M. (2017). Single chip self-tunable Ninput N-output PID control system with integrated analog front-end for miniature robotics. IEEE International Conference on Networking.

[3] Jamal, F. I., Guha, S., Eissa, M. H., Meliani, C., Ng, H. J., \& Kissinger, D., et al. (2017). A fully integrated low-power K-band chem-bio-sensor with on-chip
DC read-out in SiGe BiCMOS technology. Microwave Conference.

[4] Jamal, F. I., Guha, S., Eissa, M. H., Meliani, C., Ng, H. J., \& Kissinger, D., et al. (2017). A fully integrated low-power K-band chem-bio-sensor with on-chip DC read-out in SiGe BiCMOS technology. Microwave Conference.

[5] Zhao, N., Qian, G., Fu, X. C., Zhang, L. J., Hu, W., \& Li, R. Z., et al. (2017). Integrated optical displacement sensor based on asymmetric machzehnder interferometer chip. Optical Engineering, 56(2), 027109.

[6] Zhao, X., Yeh, C. S., Zhang, L., Lai, J. S., \& Labella, T. (2017). A 2-mhz wide-input hybrid resonant converter with ultra-compact planar coupled inductor for low power integrated on-chip applications. IEEE Transactions on Industry Applications, PP (99), 1-1.

[7] Alirezaei, I. S., Vierhaus, J., \& Burte, E. P. (2017). A monolithically integrated butt-coupled $3 \mathrm{~d}$ bulk cmos si photodetector array with a fiber couplers platform on a single-chip. Sensors \& Actuators A Physical.

[8] Li W., Zhang, H., Liu, J., Lin, J., Xue, X., \& Zhang, X., et al. (2017). On-chip high-sensitivity temperature sensor based on gain-loss coupled microresonators. Journal of the Optical Society of America B Optical Physics, 34(8), 1765.

[9] Bezuidenhout, P., Land, K., \& Joubert, T. (2017). A low-power CMOS operational amplifier IC for a heterogeneous paper-based potentiostat. Conference on Sensors.

[10] Gaggatur, J. S., Raja, I., \& Banerjee, G. (2017). OnChip Non-intrusive Temperature Detection and Compensation of a Fully Integrated CMOS RF Power Amplifier. International Conference on Vlsi Design \& International Conference on Embedded Systems.

[11] Wang, H., Britton, C., Quaiyum, F., Pullano, S. A., Taylor, L., \& Fiorillo, A. S., et al. (2018). A charge sensitive pre-amplifier for smart point-of-care devices employing polymer-based lab-on-a-chip. IEEE Transactions on Circuits \& Systems II Express Briefs, 65(8), 984-988. 